\title{
Verhaltensdispositionen II - Die psychosoziale Perspektive
}

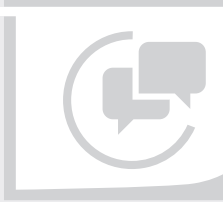

scher Natur. Wir erkennen und bestimmen sie anhand ihrer Manifestationen, die allerdings nicht ständig, sondern nur unter bestimmten Umständen auftreten. Verhaltensdispositionen lassen sich somit nur anhand ihrer situativen Manifestationen erkennen und definieren. Das Lieblingsbeispiel der philosophischen Dispositionsdiskussion - das Prädikat „Iöslich“ - zeigt auf, wie sich Dispositionsbegriffe anhand von Manifestationsbedingungen definieren lassen: $X$ ist „löslich“ bedeutet, dass $X$ sich auflöst, wenn X (unter normalen Bedingungen) in Wasser gelegt - also einer bestimmten Prozedur unterzogen - wird. Um Verhaltensdispositionen genauer zu definieren, müssen wir deren Definitionen also an eindeutige Mess- bzw. Test-Opera- tionen binden, so die Idee. Mit „Verhaltensdisposition A“ ist demnach gemeint, was ein valides A-Mess-Verfahren misst. Intelligenz etwa ist, was anerkannte Intelligenztests messen. Persönlichkeit ist, was bewährte Persönlichkeitsinventare messen und so weiter.

Die ursprünglich auf den Physiker und Nobelpreisträger Percy W. Bridgman zurückgehende Idee des „Operationalismus“ hat ihren Siegeszug in der Psychiatrie vergleichsweise spät angetreten. Dies geschah in einer Phase, in der unübersehbar geworden war, dass die, den phänomenologischen und psychodynamischen Traditionen entlehnten Terminologien relativ willkürlich und damit wenig reliabel verwendet wurden. Seit der 3. Auflage des „Diagnostical and Statistical Manual of Mental Disorders" von 1980 gelten deshalb operationale Definitionen psychischer Störungen als Maßstab: Wir verwenden Listen operational definierter Kriterien, um psychische Störungen zu definieren und entsprechend zu diagnostizieren. Eine „Major Depression " etwa ist demnach eine Verhaltensepisode von mindestens 2 Wochen, die eine Mindestanzahl an operational definierten Merkmalen aufweist - darunter „depressive Verstimmung“ oder „Verlust an Interesse oder Freude“ zuzüglich mindestens 4 weiterer Symptome [3].

losigkeit aufgegeben ${ }^{1}$. Der entscheidende Einwand besagte: Es ist abwegig, die operationale Definition eines Begriffs mit dem Referenten des Begriffs - „der Sache selbst“ - gleichzusetzen. Die erfüllten Kriterien der „Major Depression“ oder der Score, den wir mit dem Beck-Depressions-Inventar erheben, ist offenkundig nicht „die Depression“ des Patienten Müller. Vielmehr sind die Daten, die wir mittels operationaler Verfahren gewinnen allenfalls theoriebzw. verfahrensvermittelte Indikatoren für einen (!) punktuellen Zustand der Sache. Da es den logischen Empiristen um die „empirisch objektive“, allein durch neutrale Beobachtung oder Messung festgelegte Bedeutung deskriptiver Begriffe ging, ist ein zweiter Einwand von Belang: Es gilt heute als ausgemacht, dass sich die Bedeutung deskriptiver Begriffe nicht kontextfrei operational definieren lässt. Denn diese Begriffe beziehen sich nicht auf einzelne „theoriefreie Ereignis- oder Merkmalsatome“, sondern erhalten bzw. entfalten ihre Bedeutung stets in einem Kontext - im Kontext von Sätzen, im Kontext von Modellvorstellungen und Paradigmen und vor allem im Kontext etablierter wissenschaftlicher Praktiken. Die Operationalisierung abstrakter Begriffe wie „löslich“, „Neurotizismus“ oder „Depression“ kann die tatsächlichen Eigenschaften der endlos vielen, konkreten Exemplare, für die die genannten Konzepte stehen sollen, nicht erschöpfen - weder hinsichtlich der Vielgestaltigkeit der Eigenschaften noch hinsichtlich ihrer zeitlichen und kontextabhängigen Wandelbarkeit. Der ganze Ansatz krankt an der abwedie Wissenschaftsphilosophie, die sich für operationale Definitionen stark gemacht hatte, hat das Projekt bereits vor Jahrzehnten aus Gründen offensichtlicher Erfolg-
Das Verhältnis zwischen Bridgmans Operationalismus und dem Logischen Empirismus ist komplizierter als es hier dargestellt werden kann. Auf jeden Fall war Bridgman nicht mit den hier diskutierten Entwicklungen einverstanden, wie das Eingangszitat verdeutlicht. Und auch Carl Gustav Hempel und Rudolf Carnap, maßgebliche Vertreter des Logischen Empirismus, haben das Projekt einer verifikationistischen Bedeutungstheorie wissenschaftlicher Begriffe im Laufe der 1950er-Jahre unter dem Eindruck „holistischer" Argumente aufgegeben. 
gigen Vorstellung, dass ein „semantischer Atomismus “ strikter Beobachtungs- und Messprozeduren in der Lage sei, den „Atomen des physikalischen Verhaltens “ zu entsprechen.

Die Entschlossenheit, mit der die Psychiatrie im Gefolge der Verhaltenswissenschaften gleichwohl an diesem „neo-positivistischen“ Programm festhält, bildet nach meinem Dafürhalten eine maßgebliche Wurzel ihrer Sterilität und Stagnation. Unsere Forscher produzieren einen endlosen, ständig anschwellenden Strom „statistisch signifikanter Korrelationen“, die zwischen operational definierten Verhaltensparametern gefunden wurden, ohne dass dies unser Verständnis der Zusammenhänge vertieft. Die Verhaltenswissenschaften weisen, wissenschaftstheoretisch gesagt, keine „kummulative Theorieentwicklung“ auf. Der Berg an Befunden lässt die infrage stehenden Zusammenhänge nicht kognitiv transparenter werden: Wir finden weder die „Gesetze des Verhaltens" noch die „physiologischen Mechanismen“, die eine kohärente Theorie des Verhaltens ergeben könnten. Angesichts ständig neuer wissenschaftlicher Erfolgsmeldungen wird leicht übersehen, dass es sich um korrelative Befunde handelt, die früher oder später als scheinbare, als irreführende oder als nicht replizierbare Korrelationen erkannt bzw. die - weit überwiegend - einfach vergessen werden. Besonders beunruhigend ist dabei, dass sich selbst die „absolut robusten“ Befunde als „deduktiv unfruchtbar“ erweisen: Die „Verhaltenssysteme" etwa, die sich in den Lehrbüchern der Psychobiologie dargestellt finden, ermöglichen keine quantitativen Vorhersagen - weder was ihre Wechselwirkungen untereinander, noch was das Verhalten des Individuums betrifft. Die bislang gehegte Hoffnung, dass aus einem immer dichter geknüpften Netz „belastbarer“ Korrelationen ein „nomologisches Netzwerk“ erwächst, das den Weg zur schrittweisen Elaborierung der konstituierenden kausalen Zusammenhänge weist, erfüllt sich nicht. Was heute als „gesicherte Erkenntnis“ gilt, gleicht vielmehr isolierten „Puzzleteilen“, die zu einem Puzzle gehören, dessen „eigentliche Gestalt" sich nicht abzeichnet [4].

\section{Verhaltensmessung}

Der große und vielgestaltige Bereich des Messens, Detektierens und Beobachtens gehört zu den wesentlichen Aktivitäten wissenschaftlichen Erkenntnisbemühens. Dabei wird eine „empirische Domäne“ - eine Klasse von Phänomenen oder ein bestimmter Phänomen-Bereich - mit bestimmten Methoden bzw. Instrumentarien untersucht, um exakte „Daten“ zu gewinnen. Diese Daten werden unter Beachtung methodologischer Standards modelliert, um Annahmen und Hypothesen über die „verborgenen Bedingungen“ der Domäne zu prüfen. - Betrachten wir an einem Beispiel, in welchem Verhältnis Phänomene, Messung, Daten, Hypothesen und Interpretationen zu einander stehen.

Hans ist ein kluger Mann, dessen „Intelligenz" wir bestimmen wollen: Hans gilt als klug, weil er sich in vielen „kognitiv fordernden" Situationen als kompetent erwiesen hat (phänomenale Domäne). Wir erklären diese Kompetenzen mit seiner „Intelligenz“ (Dispositionshypothese). Um seine Intelligenz zu messen, lassen wir ihn 2 verschiedene Intelligenztests (Messverfahren) bearbeiten - A und B. Hans erreicht im Test A einen IQ von 120, im Test B einen IQ von 105 (Daten). Was sagen diese Befunde über Hans' Fähigkeit aus, sich in kognitiv fordernden Situationen zu bewähren? - Die folgenden Fragen sollen einschlägige Probleme des verhaltenswissenschaftlichen Operationalismus verdeutlichen.

\section{- Hat Hans 2 verschiedene Formen der Intelligenz - in Abhängigkeit von den Verfahren $A$ und $B$ ?}

Wenn Verhaltensdispositionen das sind, was die dispositionsdefinierenden Verfahren messen, dann hätte Hans 2 unterschiedliche Formen der Intelligenz. Dies ist jedoch unplausibel, weil es die realistische Intuition des dispositionsanalytischen Ansatzes verletzt: Hans Intelligenz verdankt sich einer Disposition, die sich in unterschiedlichen Situationen auf unterschiedliche Weisen manifestieren kann.

- In wieweit repräsentieren die beiden verfahrensrelativen Intelligenzen Hans' „eigentliche“ Intelligenz?
Selbst alle verfügbaren Intelligenztests zusammen sind nicht in der Lage, Hans' Intelligenz erschöpfend abzubilden. Denn Hans kann sich in sehr viel mehr Situationen als es Intelligenztest sind, auf intelligente Weise bewähren. Dabei ist zu beachten, dass Hans' Intelligenz in einem verkörperten Können, einem „knowing how“ im Sinne einer vieldimensionalen praktischen Kompetenz besteht. Dieses stillschweigende, sich im Handeln manifestierende Können lässt sich bekanntlich nicht annähernd in der Form eines expliziten, in Sätze gefassten propositionalen Wissens, einem „knowing that", artikulieren. Intelligentes, situativ angemessenes Handeln ist wie Fahrradfahren. Die Instruktion, „Setz Dich drauf! Tritt in die Pedale. Und versuch, das Gleichgewicht zu halten!“, reicht nicht aus, um dem Novizen das Fahrradfahren beizubringen. Dieser muss vielmehr so lange üben, bis die erforderlichen sensomotorischen Kompetenzen in Fleisch und Blut übergegangen sind. Dem entsprechend dürften die in der Psychiatrie beliebten Selbstadministrationsinstrumente, die psychisches Befinden in der Form von „knowing that“ abfragen, wenig zur Erfassung von handlungsleitenden Prozessen taugen.

- Wieso verhält sich Hans gelegentlich „wenig intelligent“, obwohl er erwiesenermaßen überdurchschnittlich intelligent ist?

Hans' „intelligentes“ Verhalten wird durch sehr viel mehr als durch seine „Trait“-Intelligenz bestimmt. Situative „State“-Variablen - etwa Verunsicherung oder Alkoholisierung - können eine erhebliche „intelligenzmindernde“ Rolle spielen. Hinzu kommen Wechselwirkungen zwischen den vielen verschiedenen Verhaltensdispositionen und der sich wandelnden Umwelt, in der sich Hans zu bewähren versucht.

- In welcher Beziehung steht Hans’ „eigentliche“ Intelligenz zu seiner Persönlichkeit, seiner Motivation, seinen Strategien etc.?

Die Schwierigkeiten, die diese Frage aufwirft, sind bereits im ersten Teil behandelt worden [1]. Der entscheidende Punkt meiner Argumentation ist: Das operationalis- 
tische Vorgehen versucht die offensichtlichen Unterschiede zwischen Phänomenen (Hans' intelligentem Handeln), theoriegeleiteter Methodenentwicklung (Operationalisierung), gemessenen Daten (IQ-Messwerte), deren Modellierung und Interpretation zu einem „idiotensicheren induktiven Verfahren“ zu verkürzen. Doch es gibt, wie gesagt, keine direkte, von wissenschaftlichem Handeln unabhängige Entsprechung von „Verhaltensatomen“ und „Bedeutungsatomen“, aus der sich über modellbegründete „latente Variablen“ auf das reale Geschehen schließen lässt.

\section{Operationale Beziehungen im Kontext DSM-basierter Forschung}

Überträgt man die letztgenannten Beobachtungen auf die operationalen Definitionen psychischer Störungen, so stellen sich u. a. folgende 2 Fragen:

- Wie direkt, wie robust ist die Messrelation zwischen dem operationalisierten Verhaltensmerkmal der Störungsdefinition und dem Symptom des Patienten?

Die Idee, dass es sich beim Abfragen operationaler Kriterien um eine neutrale und zuverlässige Messoperation handelt, ist abwegig. Betrachten wir dazu das Beispiel „Denkstörungen“: Denkstörungen, so die DSM-5, werden anhand der Rede des Patienten erschlossen [3]. Das bedeutet, dass der Diagnostiker über umfassendes normatives Wissen verfügen muss, um das Kriterium abzuprüfen - über ein grammatisches, semantisches und pragmatisches Wissen, dass die DSM nicht ausweist. Dies deutet an, dass wir konkrete Psychopathologien nicht Symptom für Symptom anhand eindeutig unterscheidbarer Verhaltensmerkmale analysieren. Vielmehr erfassen wir hervorstechende Anomalien im Zusammenhang eines umfassenderen Verständnisses des situierten Handelns einer Person. Dabei sind praktische Relevanzeinschätzungen von entscheidender Bedeutung.

Um den stillschweigenden „Holismus“ der Beurteilung von Denkstörungen zu verdeutlichen, mögen folgende Beobachtungen hilfreich sein: Denkstörungen fin- den sich, wohin man schaut - etwa bei so genannten „Persönlichkeitsstörungen“, „Angststörungen“ oder „Essstörungen“. Laut DSM-5 ist „Wahn“ - eine prominente Form der Denkstörungen - eine „feste Überzeugung, die trotz gegenteiliger Evidenz nicht verändert werden kann“ [3]. Doch nicht nur bei psychisch kranken Personen, sondern weit darüber hinaus finden sich häufig abwegige Denkinhalte und willkürliche Inferenzen, die „gegenteiliger Evidenz" trotzen. Warum werden diese Überzeugungen nicht als "Wahnsymptome“ bzw. als Merkmale einer „psychotischen Störung “ gewertet? Der Grund liegt auf der Hand: Wir verstehen und verwenden psychopathologische Konzepte nicht „atomistisch“, indem wir etwa die notwendigen und hinreichenden Bedingungen des Konzepts abprüfen; wir verwenden sie „prototypisch“ und „ganzheitlich“. Der klinische Novize lernt, Konzepte wie „Schizophrenie“ anhand von klaren Fällen zu gebrauchen. So werden prototypische Wissensstrukturen erworben, die es ermöglichen „holistische“ Zusammenhänge unter Salienz- und Relevanzaspekten zu erkennen und zu bewerten: Im Falle der „Schizophrenie“ greifen auffälliges Sozialverhalten, verschiedene Formen kognitiver Störungen, der Grad der Funktionsbeeinträchtigung und die Konsequenzen für das Leben des Betroffenen und seiner Umwelt ineinander. Dank dieser prototypischen Wissensstrukturen lassen sich kontextrelative „Gestalten“ erkennen und auf den Begriff bringen [5].

\section{- Was sind die Symptome, Syndrome oder Störungen, die à la DSM-5 ope- rationalisiert werden, ontologisch gesehen? Handelt es sich um wahr- nehmbare Phänomene, gemessene Daten, statistische Modellierungen oder reale Dispositionen?}

In der aktuellen Forschung geht der „semantische Atomismus “ des operationalistischen Vorgehens mit ontologischen Annahmen einher, die ich pauschalierend „Isomorphie-Annahmen“ nennen möchte: Die Symptome, die sich bei Patienten finden, entsprechen den operational definierten Merkmalen, die wir abprüfen, weil die operationale Beziehung ihre „Gleichgestaltigkeit“ garantiert: Das detektierte Phänomen bzw. „Verhaltensatom“ ist so, wie die Sache selbst ist. Die Annahme einer grundsätzli- chen „Gleichgestaltigkeit" setzt sich fort, wenn wir Syndrome - typische Gruppen gemeinsam auftretende Symptome - in den Blick nehmen: Denn die regelhafte Kovariation von Symptomen in der Form von wiedererkennbaren Syndromen spricht für eine gemeinsame kausale Ursache. Somit lautet die nächste Isomorphieannahme: Es besteht eine grundsätzliche, allerdings bislang undurchsichtige „Gleichgestaltigkeit“ zwischen Syndrom und Ätiopathogenese. Da das Nervensystem wie alle anderen Organsysteme auch nicht etwa erratisch, sondern vielmehr regelhaft arbeitet, müssen spezifische Funktions- bzw. Strukturanomalien für das Auftreten der einschlägigen Syndrome verantwortlich sein. Wenn bestimmte Verhaltensdispositionen gestört oder pathologische Verhaltensdispositionen aktiviert werden, dann kommt es zu prinzipiell vorhersagbaren klinischen Störungsbildern. Doch wie „isomorph“ sind die unbestreitbaren Gehirn-Geist-Korrespondenzen? Was, wenn verschiedene Gehirne „schizophrene“ Syndrome auf variable bzw. äquifinale Weise „generieren“? Angesichts der „explanatorischen Lücke“, die zwischen Geist und Gehirn klafft, sollten wir mit unseren ontologischen Annahmen vorsichtig sein. Für eine solche Vorsicht spricht die Beobachtung, dass sich die Anthropomorphismen, die wir auf das Nervensystem projizieren, ebenso wie viele psychologischen Universalien, die sich lange als lehrbuchwürdige Gewissheiten behauptet haben, mit der Zeit in Luft auflösen.

\section{Reliabilismus}

Ist dies nicht alles etwas spitzfindig, mag sich der erfahrene Kliniker fragen. Die Zuschreibung von Verhaltensdispositionen ist doch offensichtlich nützlich: Wir können uns innerhalb akzeptabler Fehlergrenzen auf ihre instrumentelle Rolle verlassen. Die Feststellung eines „ausgeprägten Neurotizismus“ oder einer „Minderbegabung“, wie gut oder schlecht diese auch operationalisiert sein mögen, erlaubt es doch, eine Person einzuschätzen und hinreichend verlässliche Vorhersagen über ihr Verhalten zu machen - und dass, obwohl wir die kausalen Rollen dieser Dispositionen kaum etwas wissen.

Diese pragmatische, die Reliabilität von Dispositionen betonende Sicht der Dinge 
ist Common Sense. Wir verlassen uns im Alltag wie in der Klinik auf die „Stabilität“ und die „berechenbare Nützlichkeit“ vieler mutmaßlicher Dispositionen - und dies durchaus erfolgreich. Allerdings wird leicht übersehen, was diesen praktischen Erfolg begründet. Die Kunst, Verhaltensdispositionen zu erkennen und instrumentell zuverlässig zu nutzen, ist weniger eine Frage der Bestimmung autochthoner „kausalen Potenzen“, die das Verhalten der anderen Person „von innen“ her bestimmen mögen. Sie ist vielmehr eine Sache der sozialen Interaktion bzw. des effektiven Nutzens „relationaler" sozio-kognitiver Fähigkeiten. Unsere praktische Fähigkeit, das Erleben und Verhalten anderer Personen mental „nachzubilden“, übersteigt die Möglichkeiten streng wissenschaftlicher Erklärungsansätze bei Weitem.

Wie ist diese interaktionsgestützte Fähigkeit, das verborgene Fremdpsychische zu erfassen, zu erklären? M. E. sind 2 Dimensionen unserer interpersonellen Fähigkeit, Verhaltensdispositionen zu erkennen und instrumentell zu nutzen, maßgeblich. Zudem eröffnet die interaktionistische Perspektive Möglichkeiten („Tricks“), die der methodisch kontrollierten Forschung nicht zur Verfügung stehen [6]:

Grundlage der Fähigkeit, das Seelenleben anderer zu verstehen, ist zunächst das „mimetische Mentalisieren “. Menschen sind in starkem Maße zur „interpersonellen Resonanz“ befähigt: Sie „schwingen“ gewissermaßen mit, wenn andere in „Schwingung“ geraten. Gefühle sind nicht nur ansteckend, wie das Sprichwort behauptet. Übertragene Gefühle lassen sich auf diesem Weg auch qualitativ identifizieren. Die „kausalen Potenzen“, die den Reaktionen, Gesten, Handlungen und Stimmänderungen der anderen Person zugrunde liegen mögen, übertragen sich auf den „mitfühlenden“ Beobachter, ohne dass ihm dies bewusst sein muss. Das Talent, das Fremdpsychische gewissermaßen „direkt“ interpersonell nachzubilden, scheint auf einer Passung von wahrgenommenen (fremden) und selbst gebildeten (eigenen) sensomotorischen Routinen zu beruhen. Wir erkennen am Anderen unmittelbar bzw. „zwischenkörperlich“, was wir von uns selber kennen. Ein entsprechendes Repertoire an „sensomotorischen Erfahrungsmustern" vorausgesetzt, ist es möglich, ein breites Spektrum an Verhaltensdispositionen im Verhalten des Anderen auszumachen.

Die zweite Dimension des DispositionenVerstehens wird auf der Grundlage der ersten maßgeblich sozial erlernt. Ich nenne sie „propositionales Mentalisieren“, weil sie die wahrgenommenen Verhaltensweisen auf den konventionellen Begriff bzw. Satz zu bringen erlaubt. Wer propositional mentalisiert, der erkennt beobachtbare Verhaltensepisoden als Handlungen aufgrund (unbeobachtbarer) mentaler Prozesse eines bestimmten, sprachlich fassbaren Inhalts. Wenn wir auf diese Weise Wünsche und Überzeugungen, Charaktereigenschaften, Denkstile und Bewältigungsstrategien am Werke sehen, dann spielen wir über die Bande konventioneller psychologischer Diskurse: Wir verwenden die Alltagssprache in der Form geläufiger, intersubjektiv verständlicher Erklärungsmuster, um das Fremdpsychische zu verstehen. Wenn Hans etwa erklärt, er wolle sich zur Ruhe setzen, dann lassen sich viele plausible Szenarien bemühen, um zu verstehen, warum er vorzeitig in den Ruhestand geht oder was er sich von diesem Schritt erhofft. Den Stoff, aus dem diese Szenarien gemacht sind, liefert eine immense virtuelle Matrix aus möglichen Verhaltensdispositionen, einschlägigen „Handlungsregeln“ und narrativen Ressourcen, die dem Interpreten endlose inferenzielle Möglichkeiten eröffnen. Wer etwa unseren Hans näher kennt, der wird seine Intelligenz deswegen nicht als nur „eine Disposition“, sondern als ein zusammenhängendes System aus Überzeugungen und Fähigkeiten, einem bestimmten Denk- bzw. Inferenzstil und diversen psychischen („Persönlichkeit“), sozialen („Beziehungen“) und institutionellen Besonderheiten (Beruf, Status, Leistungen, Rollen) und vielem mehr verstehen. Zusammen mit den „mimetischen Eindrücken“ aus unmittelbaren Interaktionen ermöglicht dieses System „propositionaler Wissensmarken“ ein dynamisches „Framing“ des psychologischen Geschehens: Informationen unterschiedlicher Arten und Quellen werden kontextsensitiv gewichtet, fortlaufend aktualisiert und über längere Beobachtungszeiträume getestet, um möglichst kohärente Hypothesen zu generieren. Diese Form der abduktiven „inference to the best explanation" unterscheidet sich in verschie- dener Hinsicht grundlegend vom wissenschaftlichen Vorgehen, wie die folgenden „Tricks“ zeigen mögen [7].

Der erste „Trick“ besteht in der Rolle fortlaufender Interaktion: Ich, der psychologisierende Beobachter, bin Teil der Situation. Ich gestalte diese mit, was es ermöglicht, die Verhaltensdispositionen des Anderen fortlaufend „experimentell“ zu sondieren. Meine „Messungen“ sind nicht punktförmig, sondern prozessartig. Die resultierenden Dispositionshypothesen basieren auf ökologisch valideren „Daten“, weil sie fortlaufend „upgedated “ und revidiert werden und dank „kontextsensibler Testung“ präziser sein können. Ich bin nicht auf die „Schnappschuss-Ontologie“ punktueller Messoperationen und nicht auf wenige operationalisierte Variablen beschränkt, sondern kann das mentale Geschehen des Anderen als Prozess nachbilden.

Der zweite „Trick“ besteht in der Flexibilität der Interpretation. Wenn sich meine Vorhersagen aufgrund bisheriger Interaktionserfahrungen als zu ungenau erweisen, kann ich ad hoc Zusatzhypothesen einführen oder den Interpretationsrahmen wechseln. Anders als der Verhaltenswissenschaftler „testet“ der intuitive Psychologe viele Hypothesen gleichzeitig, um ein möglichst kohärentes Gesamtergebnis zu erreichen. Spezifische Einzelthesen, die es empirisch-kritisch zu testen gilt, sind seine Sache nicht.

Zum Dritten schließlich finden meine dispositionsbasierten Verhaltensvorsagen oft im Rahmen interpersoneller Aushandlungsprozesse statt. Wir können uns im Gespräch darüber verständigen, was Sache ist. Insofern kann meine Zuschreibung und Abschätzung von Dispositionen durch die Angaben des Betroffenen erleichtert oder gar bestätigt werden. Wenn ich und der andere dahingehend übereinstimmen, was Präsident Trump oder Boris Johnson für „Typen“ sind, dann verknüpfen sich unsere „Überzeugungssysteme“ auf eine Weise, die die weitere Verhaltenskoordination in verschiedener Hinsicht erleichtern kann. Das Bemerkenswerte an diesem „unwissenschaftlichen“, auf interpersonelle Verständigungs- und Aushandlungsprozesse abstellenden Ansatz ist m. E., dass dieser Hinweise zur Lösung der diskutierten Pro- 
bleme des Verhaltensdispositionalismus liefern kann:

Das Problem des Doktors aus Molières Stück ist in der lebensweltlichen Praxis praktisch keines [1]: Die problematisierte Phänomen-Trait-Ambiguität „verschwindet“" in der Unschärfe des interpersonellen Verständigungsprozesses. Die hartnäckigen Schwierigkeiten, Verhaltensdispositionen auf die Aktivität bestimmter physiologische Mechanismen oder „Rechenprozesse in kognitiven Architekturen“ zurückzuführen, betreffen den Alltagspsychologen ebenfalls nicht [1]. Dieser beschränkt sich auf ein „externalistisches“ Vorgehen, in dem er das beobachtete Verhalten mental nachbildet und das verborgene mentale Geschehen fortlaufend simulierend approximiert. Dies geschieht - anders als in den Verhaltenswissenschaften - auf der Grundlage einer normativen, d. h. arbiträren Matrix „plausibler Handlungserklärungen“.

Der Reliabilismus dieser alltagspsychologischen Dispositionsanalysen vermeidet die Sackgasse des Operationalismus. Die Daten bestehen nicht in operationalisierten „Punkt-Messungen“, sondern in fort- laufenden Interaktions- und Simulationsprozessen. Sie betreffen nicht eine Eigenschaft oder Dimension des Verhaltens, sondern alle, die dem Beobachter zugänglich sind. M. E. zeigt die Rekonstruktion des Reliabilismus den Weg einer potentiell fruchtbareren, die Fähigkeiten kompetenter Alltagspsychologen rekonstruierender Forschung auf.

\section{Interessenkonflikt}

Es bestehen keine Interessenkonflikte.

\section{Korrespondenzadresse}

Dr. Markus R. Pawelzik

EOS-Klinik für Psychotherapie

Hammerstr. 18

48153 Münster

m.pawelzik@alexianer.de
Literatur

[1] Pawelzik M. Verhaltensdispositionen I: Die biologische Perspektive. Nervenheilunde 2019; 39: 965-969

[2] Bridgman PW. The present state of operationalism. In: P. Frank. (Ed.) The validation of scientific theories. New York: Collier; 1961

[3] Falkai P, Wittchen H-U. Hrsg. Diagnostisches und Statistisches Manual Psychischer Störungen. Göttingen: Hogrefe; 2015

[4] Meehl P. Theoretical risks and tabula asterisks: Sir Karl, Sir Ronald and the slow progress of soft psychology. In: N. G. Weller et al. (Eds.) A Paul Meehl Reader. Essays on the practice of scientific psychology. Mahwah: Lawrence Earlbaum Associates; 2006

[5] Murphy GL. The big book of concepts. Cambridge, Massachusetts: The MIT Press; 2002

[6] Pawelzik M. On two kinds of mentalizing (in preparation)

[7] Lipton P. Inference to the best explanation. London: Routledge; 1991

Bibliografie

DOI https://doi.org/10.1055/a-0976-0583 Nervenheilkunde 2020; 39: 93-97 (c) Georg Thieme Verlag KG Stuttgart · New York ISSN 0722-1541 